\title{
LIMIT THEOREMS FOR A GENERALIZED ST PETERSBURG GAME
}

\author{
ALLAN GUT, ${ }^{*}$ Uppsala University
}

\begin{abstract}
The topic of the present paper is a generalized St Petersburg game in which the distribution of the payoff $X$ is given by $\mathrm{P}\left(X=s r^{(k-1) / \alpha}\right)=p q^{k-1}, k=1,2, \ldots$, where $p+q=1$, $s=1 / p, r=1 / q$, and $0<\alpha \leq 1$. For the case in which $\alpha=1$, we extend Feller's classical weak law and Martin-Löf's theorem on convergence in distribution along the $2^{n}$-subsequence. The analog for $0<\alpha<1$ turns out to converge in distribution to an asymmetric stable law with index $\alpha$. Finally, some limit theorems for polynomial and geometric size total gains, as well as for extremes, are given.
\end{abstract}

Keywords: St Petersburg game; sums of i.i.d. random variables; Feller's weak law of large numbers; domains of attraction; convergence along subsequences; extremes; stable law; slow variation; regular variation

2010 Mathematics Subject Classification: Primary 60F05; 60G50

Secondary $26 \mathrm{~A} 12$

\section{Introduction}

The original St Petersburg game is defined as follows: toss a fair coin repeatedly until a head appears. If this happens at trial number $k$, you receive $2^{k}$ Euros. A natural fair price for you to participate would be the expected value, which, however, as is well known, is infinite, since the random variable $X$ describing the payoff is

$$
\mathrm{P}\left(X=2^{k}\right)=\frac{1}{2^{k}}, \quad k=1,2, \ldots
$$

One variant of the game is to set the fee as a function of the number of games, which leads to the celebrated Feller solution [6], namely, that if $X, X_{1}, X_{2}, \ldots$ are independent and identically distributed (i.i.d.) random variables as above, and $S_{n}=\sum_{k=1}^{n} X_{k}, n \geq 1$, then

$$
\frac{S_{n}}{n \log _{2} n} \stackrel{\mathrm{P}}{\rightarrow} 1 \quad \text { as } n \rightarrow \infty,
$$

where $\log _{2}(\cdot)$ denotes the logarithm relative to base 2 (similarly, $\log _{\eta}(\cdot)$ below denotes the logarithm relative to base $\eta>0$ ), and ' $\stackrel{P}{\rightarrow}$ ' denotes convergence in probability. For details, see [7, Chapter X] and [8, Chapter VII] (and/or [10, Section 6.4.1]). More on the history of the game can be found in [13].

The present paper is devoted to the generalization in which a biased coin for which $\mathrm{P}($ head $)=p, 0<p<1$, is tossed repeatedly until a head appears. If this happens at

Received 14 December 2009; revision received 7 May 2010.

* Postal address: Department of Mathematics, Uppsala University, Box 480, SE-751 06 Uppsala, Sweden.

Email address: allan.gut@math.uu.se 
trial number $k$, you receive $s r^{(k-1) / \alpha}$ Euros, where $s=p^{-1}, r=q^{-1}=(1-p)^{-1}$, and $\alpha>0$. This, in turn, induces the random variable

$$
\mathrm{P}\left(X=s r^{(k-1) / \alpha}\right)=p q^{k-1}, \quad k=1,2, \ldots
$$

Since in the classical game the expected payoff is infinite and we wish to retain this situation here, we confine ourselves in the following to the case in which $0<\alpha \leq 1$ (except for some short remarks).

Remark 1.1. Authors who have studied generalized St Petersburg games have, throughout, considered the case when the payoff $X$ follows the distribution

$$
\mathrm{P}\left(X=r^{k / \alpha}\right)=p q^{k-1}, \quad k=1,2, \ldots
$$

Our choice of payoff preserves the fairness of the game, in that the expected gain after one round equals $p(s-1)+q(-1)=0$ (under the model assumption that the entrance fee remains 1 Euro).

The case in which $p=q=\frac{1}{2}$ and $\alpha=1$ reduces, of course, to the classical game. However, whereas we still obtain a weak law for general $p$ when $\alpha=1$, it turns out, as we shall see, that the behaviour for $0<\alpha<1$ is drastically different in that we obtain convergence in distribution to a stable law. This is the content of our first result.

As for convergence in distribution in the classical case, Martin-Löf, in his famous paper [13], obtained convergence in distribution to an infinitely divisible distribution along the geometric subsequence $2^{n}$. Our second result extends Martin-Löf's theorem to the general case with $\alpha=1$.

The results mentioned so far, together with a limit theorem for the 'maximal payoff so far', are stated in Section 2 and proved in Sections 4 and 5, respectively, after some preliminaries in Section 3. In Section 6 we consider the problem of moderate and large gains, which are somewhat related to large deviations. A final section contains some additional remarks.

\section{Main results}

Throughout, let $X, X_{1}, X_{2}, \ldots$ be i.i.d. random variables distributed according to

$$
\mathrm{P}\left(X=s r^{(k-1) / \alpha}\right)=\mathrm{P}\left(X=p^{-1} q^{-(k-1) / \alpha}\right)=p q^{k-1}, \quad k=1,2, \ldots,
$$

where $0<p=1-q<1, s=1 / p, r=1 / q$, and $0<\alpha \leq 1$, and set $S_{n}=\sum_{k=1}^{n} X_{k}$ and $M_{n}=\max _{1 \leq k \leq n} X_{k}, n \geq 1$.

Theorem 2.1. (i) If $\alpha=1$ then

$$
\frac{S_{n}}{n \log _{1 / q} n} \stackrel{\mathrm{P}}{\rightarrow} 1 \text { as } n \rightarrow \infty .
$$

(ii) If $0<\alpha<1$ then

$$
p^{3 / \alpha-1}\left(q^{1-2 / \alpha}-1\right)^{1 / \alpha} \frac{S_{n}}{n^{1 / \alpha}} \stackrel{\mathrm{D}}{\rightarrow} Y_{\alpha} \quad \text { as } n \rightarrow \infty,
$$

where $Y_{\alpha}$ is a positive random variable with a stable $\alpha$-distribution, whose Laplace transform equals $\exp \left\{-t^{\alpha}\right\}, t>0$, and $\stackrel{\mathrm{D}}{\rightarrow}$ ' denotes convergence in distribution. 
(iii) For $0<\alpha \leq 1$,

$$
\frac{p M_{n}}{n^{1 / \alpha}} \stackrel{\mathrm{D}}{\rightarrow} \Phi_{\alpha} \quad \text { as } n \rightarrow \infty
$$

where $\Phi_{\alpha}(x)=\exp \left\{-x^{-\alpha}\right\}, x>0$, the Fréchet distribution.

Remark 2.1. For $p=q=\frac{1}{2}$ and $\alpha=1$, Theorem 2.1(i) reduces to (1.1).

Remark 2.2. For distribution (1.3), Kevei [12] stated the result in Theorem 2.1(i) without proof; in that setting the limit equals $p / q$. Adler and Rosalsky [2, Theorem 4] proved a weak law for the weighted sum $\sum_{k=1}^{n} k^{\gamma} X_{k}$, where $\gamma>-1$.

As we can see in Theorem 2.1(i) and (ii), the results for the two cases are generically different for $\alpha=1$ and $0<\alpha<1$, respectively. In the former case we have convergence in probability, whereas in the latter we have convergence in distribution. Moreover, the normalizations are slightly different in that an additional logarithm is needed in (i).

First of all, additional logarithms are frequently needed in cases of an ' $\alpha=1$ situation'. Typically, this is due to the fact that $\sum_{k=1}^{n} 1 / k^{\alpha}$ grows like $n^{1-\alpha}$ when $\alpha<1$, but logarithmically when $\alpha=1$; the harmonic series causes a lot of trouble for mathematicians. In the present case this pertains to the truncated first moment $\mathrm{E} X \mathbf{1}\{X \leq x\}$, as is found in (3.2), below.

Another interesting point is that we obtain convergence in distribution for the full sequence in Theorem 2.1 when $0<\alpha<1$, whereas in the classical case ( $p=q=\frac{1}{2}$ and $\alpha=1$ ) we obtain convergence in distribution only along the subsequence $2^{n}$, as was shown by Martin-Löf [13].

Our next result completes the case in which $\alpha=1$, in that we provide a generalization of the first part of Martin-Löf's theorem.

Theorem 2.2. Let $N=q^{-n}$. Then

$$
\frac{S_{N}-N \log _{1 / q} N}{N}=\frac{S_{N}}{N}-\log _{1 / q} N=\frac{S_{N}}{N}-n \stackrel{\mathrm{D}}{\rightarrow} Z,
$$

where $Z$ is defined via the characteristic function $\varphi_{Z}(t)=E \exp \{\mathrm{i} t Z\}$ given by

$$
\varphi_{Z}(t)=\exp \left\{\sum_{k=-\infty}^{-1}\left(\exp \left\{\mathrm{i} t p^{-1} q^{-k}\right\}-1-\mathrm{i} t p^{-1} q^{-k}\right) p q^{k}+\sum_{k=0}^{\infty}\left(\exp \left\{\mathrm{i} t p^{-1} q^{-k}\right\}-1\right) p q^{k}\right\} .
$$

Remark 2.3. In complete analogy with [13] we infer that the limit law is infinitely divisible, that the corresponding Lévy measure has point masses $p q^{k}$ at the points $p^{-1} q^{-k}$ for $k \in \mathbb{Z}$, and that we are facing a compound Poisson distribution.

By replacing $2^{m}$ with $q^{-m}=r^{m}$ in the proof of [13, Theorem 2] we verify that the limit distribution is semistable and satisfies the scaling law

$$
\log \varphi_{Z}\left(q^{-m} t\right)=q^{-m}\left(\log \varphi_{Z}(t)-\mathrm{i} m t\right) \quad \text { for } m \in \mathbb{Z}
$$

This also illustrates the fact that we do not have a limit distribution for the full sequence (since such a limit would have been stable with index 1). 


\section{Preliminaries}

In this section we collect some facts that will be used later with or without specific reference.

Lemma 3.1. For $X$ as defined in Theorem 2.1, we have

$$
\mathrm{E} X=\left\{\begin{array}{ll}
\frac{q^{1 / \alpha}}{q^{1 / \alpha}-q} & \text { for } \alpha>1, \\
\infty & \text { for } \alpha \leq 1,
\end{array} \quad \mathrm{E} X^{\beta}= \begin{cases}\frac{p^{1-\beta} q^{\beta / \alpha}}{q^{\beta / \alpha}-q} & \text { for } 0<\beta<\alpha \leq 1, \\
\infty & \text { for } \beta \geq \alpha\end{cases}\right.
$$

Moreover, as $x \rightarrow \infty$,

$$
\begin{aligned}
\mathrm{P}(X>x) & \sim\left(\frac{x}{p}\right)^{-\alpha} \text { for } \alpha>0, \\
\mathrm{E} X \mathbf{1}\{X \leq x\} & \sim \begin{cases}\log _{1 / q} x & \text { for } \alpha=1, \\
\frac{q^{1 / \alpha}}{p^{1-\alpha}\left(q-q^{1 / \alpha}\right)} x^{1-\alpha} & \text { for } 0<\alpha<1,\end{cases}
\end{aligned}
$$

and

$$
\text { E } X^{2} \mathbf{1}\{X \leq x\} \sim \begin{cases}\frac{q}{p^{3}} x & \text { for } \alpha=1 \\ \frac{q^{2 / \alpha}}{p^{3-\alpha}\left(q-q^{2 / \alpha}\right)} x^{2-\alpha} & \text { for } 0<\alpha<1 .\end{cases}
$$

Proof. Upon noting that

$$
\begin{gathered}
\mathrm{E} X^{\beta}=\sum_{k=1}^{\infty} s r^{(k-1) \beta / \alpha} p q^{k-1}, \\
\mathrm{E} X=\sum_{k=1}^{\infty} s r^{(k-1) / \alpha} p q^{k-1}, \\
\mathrm{P}(X>x) \sim \sum_{k>\alpha \log _{r} x+\alpha \log _{r} s+1} p q^{k-1}, \\
\mathrm{E} X \mathbf{1}\{X \leq x\} \sim \sum_{1 \leq k \leq \alpha \log _{r} x+\alpha \log _{r} s+1} s r^{(k-1) / \alpha} p q^{k-1}, \\
\sum_{1 \leq k \leq \alpha} s^{2} r^{2(k-1) / \alpha} p q^{k-1},
\end{gathered}
$$

the conclusions follow via elementary computations.

We also need the concepts of regular variation and slow variation.

Definition 3.1. Let $a>0$. A positive measurable function $u$ on $[a, \infty)$ varies regularly at $\infty$ with exponent $\rho,-\infty<\rho<\infty$, denoted by $u \in \mathcal{R V}(\rho)$, if and only if

$$
\frac{u(t x)}{u(t)} \rightarrow x^{\rho} \quad \text { as } t \rightarrow \infty \text { for all } x>0 .
$$

If $\rho=0$, the function is slowly varying at $\infty$, denoted by $u \in \delta \mathcal{V}$. 
For more on this, we refer the reader to [3], and, for a quick summary of some basic facts and relations, we refer the reader to [10, Section A.7].

\section{Proof of Theorem 2.1}

Proof of (i). We first observe that the function $x \log _{1 / q} x \in \mathcal{R V}(1)$.

Next, since, by (3.1),

$$
n \mathrm{P}\left(X>n \log _{1 / q} n\right) \sim \frac{1}{\log _{1 / q} n} \rightarrow 0 \quad \text { as } n \rightarrow \infty,
$$

and, by (3.2),

$$
n \mathrm{E} X \mathbf{1}\left\{X \leq n \log _{1 / q} n\right\} \sim n\left(\log _{1 / q} n+\log _{1 / q} \log _{1 / q} n\right) \sim n \log _{1 / q} n \quad \text { as } n \rightarrow \infty,
$$

the conclusion is an immediate consequence of the extension of Feller's weak law of large numbers given in [9, Theorem 1.3]; cf. also [10, Theorem 6.4.2].

Proof of (ii). In view of (3.3) it follows from standard criteria for domains of attraction (see, e.g. [8, Chapter IX], and/or [10, Section 9.3]) that the distribution of $X$ belongs to the domain of attraction of a positive stable law with index $\alpha$.

Moreover, the normalization $a_{n}$ is defined as

$$
a_{n}=\inf \left\{x: \frac{n U(x)}{x^{2}} \leq 1\right\}
$$

where $U(x)=\mathrm{E} X^{2} \mathbf{1}\{X \leq x\}$.

In the present case this means that we have to solve the equation

$$
\frac{n}{x^{2}} \frac{q^{2 / \alpha}}{p^{3-\alpha}\left(q-q^{2 / \alpha}\right)} x^{2-\alpha}=1,
$$

which entails

$$
a_{n}=\left(\frac{n q^{2 / \alpha}}{p^{3-\alpha}\left(q-q^{2 / \alpha}\right)}\right)^{1 / \alpha}=\left(\frac{q^{2 / \alpha}}{p^{3-\alpha}\left(q-q^{2 / \alpha}\right)}\right)^{1 / \alpha} n^{1 / \alpha} .
$$

Proof of (iii). It follows from standard criteria for domains of attraction to extreme value distributions that $X$ belongs to the domain of attraction of the Fréchet distribution, and that the normalization may be chosen to be

$$
a_{n}=\inf \left\{x: 1-F(x) \leq \frac{1}{n}\right\}
$$

cf., e.g. [10, Theorem 9.6.3]. In the present case this amounts (via (3.1)) to solving the equation $(x / p)^{-\alpha}=1 / n$. The conclusion follows.

\section{Proof of Theorem 2.2}

Theorem 2.2 is proved via a fairly straightforward modification of the corresponding proof in [13]. 
To this end, we first recall that $s=1 / p, r=1 / q$, and, hence, that $N=r^{n}$. We thus wish to show that

$$
\varphi_{Z}(t)=\exp \left\{\sum_{k=-\infty}^{-1}\left(\mathrm{e}^{\mathrm{i} t s r^{k}}-1-\mathrm{i} t s r^{k}\right) p q^{k}+\sum_{k=0}^{\infty}\left(\mathrm{e}^{\mathrm{i} t s r^{k}}-1\right) p q^{k}\right\} .
$$

Now, $\mathrm{P}\left(X=s r^{k-1}\right)=p q^{k-1}$, so that

$$
\varphi_{X}(t)=\mathrm{Ee}^{\mathrm{i} t X}=\sum_{k=1}^{\infty} \mathrm{e}^{\mathrm{i} t s r^{k-1}} p q^{k-1}
$$

from which it follows that

$$
\begin{aligned}
\varphi_{S_{N} / N-n}(t) & =\mathrm{e}^{-\mathrm{i} t n}\left(\sum_{k=0}^{\infty} \mathrm{e}^{\mathrm{i} t s r^{k} / N} p q^{k}\right)^{N} \\
& =\mathrm{e}^{-\mathrm{i} t n}\left(\sum_{k=0}^{\infty} \mathrm{e}^{\mathrm{i} t s r^{k-n}} p q^{k}\right)^{N} \\
& =\mathrm{e}^{-\mathrm{i} t n}\left(1+\sum_{k=0}^{\infty}\left(\mathrm{e}^{\mathrm{i} t s r^{k-n}}-1\right) p q^{k}\right)^{N} \\
& =\mathrm{e}^{-\mathrm{i} t n}\left(1+q^{n} \sum_{k=-n}^{\infty}\left(\mathrm{e}^{\mathrm{i} t s r^{k}}-1\right) p q^{k}\right)^{N} \\
& =\mathrm{e}^{-\mathrm{i} t n}\left(1+\frac{1}{N} \sum_{k=-n}^{\infty}\left(\mathrm{e}^{\mathrm{i} t s r^{k}}-1\right) p q^{k}\right)^{N} \\
& =\mathrm{e}^{-\mathrm{i} t n}\left(1+\frac{1}{N} \sum_{k=-n}^{-1}\left(\mathrm{e}^{\mathrm{i} t s r^{k}}-1-\mathrm{i} t s r^{k}\right) p q^{k}+\mathrm{i} t \frac{n}{N}\right. \\
& \left.\quad+\frac{1}{N} \sum_{k=1}^{\infty}\left(\mathrm{e}^{\mathrm{i} t s r^{k}}-1\right) p q^{k}\right)^{N} \\
& \left.\left.=\mathrm{e}^{-\mathrm{i} t n\left(1+\frac{1}{N}\left\{\sum_{k=-n}^{-1}\left(\mathrm{e}^{\mathrm{i} t s r^{k}}-1-\mathrm{i} t s r^{k}\right) p q^{k}+\mathrm{i} t n\right.\right.}\left(\mathrm{e}^{\mathrm{i} t s r^{k}}-1\right) p q^{k}\right\}\right)^{N},
\end{aligned}
$$

which converges to $\varphi_{Z}(t)$ as given in (5.1) as $n \rightarrow \infty$.

\section{Polynomial and geometric size deviations}

Another kind of problem that has been investigated earlier is that of moderate or large gains.

As for polynomial size deviations, Theorem 2.1 and Corollary 2.3 of $\mathrm{Hu}$ and Nyrhinen [11]—which concern more general distributions with regularly varying tails-adapted to the St Petersburg setting provide the following result. 
Theorem 6.1. For any $b>1 / \alpha$,

$$
\lim _{n \rightarrow \infty} \frac{\log _{1 / q} \mathrm{P}\left(S_{n}>n^{b}\right)}{\log _{1 / q} n}=1-\alpha b, \quad \lim _{n \rightarrow \infty} \frac{\log _{1 / q} \mathrm{P}\left(M_{n}>n^{b}\right)}{\log _{1 / q} n}=1-\alpha b .
$$

Proof. The only thing to check is, in the notation of [11], that $\bar{\alpha}=\underline{\alpha}$ in Equations (5) and (6) of [11].

Now, by (3.1),

$$
\mathrm{P}(\log X>x)=\mathrm{P}\left(X>\mathrm{e}^{x}\right) \sim\left(\frac{\mathrm{e}^{x}}{p}\right)^{-\alpha},
$$

so that

$$
\frac{\log \mathrm{P}(\log X>x)}{x} \sim \frac{\alpha \log p}{x}-\alpha \sim-\alpha \quad \text { as } x \rightarrow \infty,
$$

after which the claims follow from [11, Theorem 2.1 and Corollary 2.3].

Remark 6.1. Theorem 6.1 is stated for natural logarithms in [11], which, of course, does not change anything.

Turning to geometric size deviations, Stoica [14] provided a result for the classical St Petersburg game to the effect that, for any $\varepsilon>0$ and $b>1$,

$$
\log _{2} \mathrm{P}\left(S_{n}>\varepsilon b^{n}\right) \sim \log _{2} \mathrm{P}\left(M_{n}>\varepsilon b^{n}\right) \sim-n \log _{2} b \quad \text { as } n \rightarrow \infty .
$$

As a corollary, it was shown that the same asymptotics also hold for the maximal gain (something that, alternatively, can be seen with the aid of relation (6.4) below).

The following result extends Theorem 1 and Corollary 1 of [14]. The proof follows in parts closely to that given in [14].

Theorem 6.2. For any $\varepsilon>0$ and $b>1$,

$$
\begin{aligned}
& \lim _{n \rightarrow \infty} \frac{\log _{1 / q} \mathrm{P}\left(X>\varepsilon b^{n / \alpha}\right)}{\log _{1 / q}\left(\varepsilon b^{n / \alpha}\right)}=-\alpha, \\
& \lim _{n \rightarrow \infty} \frac{\log _{1 / q} \mathrm{P}\left(S_{n}>\varepsilon b^{n / \alpha}\right)}{n}=-\frac{\log _{1 / q} b}{\alpha}, \\
& \lim _{n \rightarrow \infty} \frac{\log _{1 / q} \mathrm{P}\left(M_{n}>\varepsilon b^{n / \alpha}\right)}{n}=-\frac{\log _{1 / q} b}{\alpha} .
\end{aligned}
$$

In particular, for $b=1 / q=r$, the limits in (6.2) and (6.3) equal $-1 / \alpha$.

Proof. Relation (6.1) is an immediate consequence of (3.1), according to which

$$
\log _{1 / q} \mathrm{P}\left(X>\varepsilon b^{n / \alpha}\right) \sim-\alpha\left(\log _{1 / q}\left(\varepsilon b^{n / \alpha}\right)-\log _{1 / q} p\right) \quad \text { as } n \rightarrow \infty .
$$

As for (6.3), we exploit [10, Lemma 4.2] (i.e. [9, Lemma 2.2], also cited as [14, Lemma 1]), to conclude, as in $[10$, p. 270$]$, that

$$
\frac{1}{2} n \mathrm{P}\left(X>\varepsilon b^{n / \alpha}\right) \leq \mathrm{P}\left(M_{n}>\varepsilon b^{n / \alpha}\right) \leq n \mathrm{P}\left(X>\varepsilon b^{n / \alpha}\right) \text { for large } n,
$$

which, after taking logarithms, dividing by $n$, letting $n \rightarrow \infty$, and appealing to (6.1), proves the conclusion for $M_{n}$. 
Finally, for $S_{n}$, we obtain a lower bound from the trivial fact that $S_{n} \geq M_{n}$, viz.

$$
\liminf _{n \rightarrow \infty} \frac{\log _{1 / q} \mathrm{P}\left(S_{n}>\varepsilon b^{n / \alpha}\right)}{n} \geq-\frac{\log _{1 / q} b}{\alpha} .
$$

The upper bound follows by copying the lower half of page 565 of [14], replacing $b^{n}$ by $b^{n / \alpha}$ at appropriate places. We omit the details.

\section{Some remarks}

We close with some additional comments. In Subsection 7.1 we discuss the possibility of having almost-sure convergence in Theorem 2.1(i), and in Subsection 7.2 we state two easy consequences for the logarithm of the payoff.

\subsection{Almost-sure convergence?}

The obvious question that comes to mind is whether or not there is almost-sure convergence in Theorem 2.1(i). Now, since $\mathrm{E} X=+\infty$ when $\alpha=1$, the converse of the Kolmogorov strong law tells us that this cannot be the case. However, more can be said. Namely, by (3.1) we have

$$
\sum_{n=1}^{\infty} \mathrm{P}\left(X>c n \log _{1 / q} n\right)=\infty \quad \text { for any } c>0,
$$

so that, by the first Borel-Cantelli lemma,

$$
\mathrm{P}\left(X_{n}>c n \log _{1 / q} n \text { infinitely often }\right)=1 \text { for any } c>0,
$$

which, upon noting that $S_{n} \geq X_{n}$ for all $n \geq 1$, tells us that

$$
\limsup _{n \rightarrow \infty} \frac{S_{n}}{n \log _{1 / q} n}=\infty \text {. }
$$

For the classical game ( $p=q=\frac{1}{2}$ ), this was first established in [4].

As for the limit inferior, it follows from Example 4 of [1] (with $X$ as in (1.3)—cf. also [2, Theorem 4] and [5, Equation (3.9)]) that

$$
\liminf _{n \rightarrow \infty} \frac{S_{n}}{n \log _{1 / q} n}=1 .
$$

For completeness, we mention that the mean $\mathrm{E} X$ is finite whenever $\alpha>1$, as a consequence of which the Kolmogorov strong law immediately tells us that

$$
\frac{S_{n}}{n} \stackrel{\text { a.s. }}{\longrightarrow} \mathrm{E} X=\frac{q^{1 / \alpha}}{q^{1 / \alpha}-q} \quad \text { as } n \rightarrow \infty,
$$

and that the variance is finite whenever $\alpha>2$, in which case we are facing a central limit problem. Finally, for the boundary case, $\alpha=2$, the truncated second moment

$$
\mathrm{E} X^{2} \mathbf{1}\{X \leq x\} \in \& \mathcal{V},
$$

which implies that $X$ belongs to the domain of attraction of the normal distribution. 


\subsection{The logarithm of the payoff}

Section 6 provides results on asymptotics of the logarithm of the tail probability of the payoff. Another, much more trivial, task is to consider the logarithm of the payoff itself. Namely, since $\log _{2} X$ has a geometric distribution with mean 2 in the classical case, it follows immediately, by the strong law of large numbers, that

$$
\frac{1}{n} \sum_{k=1}^{n} \log _{2} X_{k} \stackrel{\text { a.s. }}{\longrightarrow} 2 \text { as } n \rightarrow \infty \text {. }
$$

Since, for $0<\alpha<1$,

$$
\mathrm{E}\left(\log _{1 / q} X\right)=\sum_{k=1}^{\infty}\left(\log _{r} s+\frac{k-1}{\alpha}\right) p q^{k-1}=\log _{r} s+\frac{1}{\alpha} \sum_{k=0}^{\infty} k p q^{k}=-\log _{1 / q} p+\frac{1}{\alpha} \frac{q}{p},
$$

we find, in complete analogy with (7.1), that

$$
\frac{1}{n} \sum_{k=1}^{n} \log _{1 / q} X_{k} \stackrel{\text { a.s. }}{\longrightarrow} \frac{q}{\alpha p}-\log _{1 / q} p \quad \text { as } n \rightarrow \infty
$$

in that case.

\section{Acknowledgements}

The point of departure of this paper was the presentation by, and some discussions with, Gyula Pap during the EMS meeting in Toulouse 2009. I also wish to thank Anders Martin-Löf for some stimulating conversations, in particular for the remark on the fairness of the game mentioned in Remark 1.1, which led me to consider the payoff distribution (1.2) rather than that of (1.3). Finally, I would like to thank the anonymous referee for a careful reading of the manuscript, in particular for suggesting some clarifications.

\section{References}

[1] AdLer, A. (1990). Generalized one-sided laws of the iterated logarithm for random variables barely with or without finite mean. J. Theoret. Prob. 3, 587-597.

[2] Adler, A. And Rosalsky, A. (1989). On the Chow-Robbins 'fair' games problem. Bull. Inst. Math. Acad. Sinica 17, 211-227.

[3] Bingham, N. H., Goldie, C. M. And Teugels, J. L. (1987). Regular Variation. Cambridge University Press.

[4] Chow, Y. S. and Robbins, H. (1961). On sums of independent random variables with infinite moments and 'fair' games. Proc. Nat. Acad. Sci. USA 47, 330-335.

[5] Csörgő, S. And Simons, G. (1996). A strong law of large numbers for trimmed sums, with applications to generalized St. Petersburg games. Statist. Prob. Lett. 26, 65-73.

[6] Feller, W. (1945). Note on the law of large numbers and 'fair' games. Ann. Math. Statist. 16, 301-304.

[7] Feller, W. (1968). An Introduction to Probability Theory and Its Applications, Vol 1, 3rd edn. John Wiley, New York.

[8] Feller, W. (1971). An Introduction to Probability Theory and Its Applications, Vol 2, 2nd edn. JohnWiley, New York.

[9] Gut, A. (2004). An extension of the Kolmogorov-Feller weak law of large numbers with an application to the St. Petersburg game. J. Theoret. Prob. 17, 769-779.

[10] Gut, A. (2007). Probability: A Graduate Course. Springer, New York.

[11] Hu, Y. and Nyrhinen, H. (2004). Large deviations view points for heavy-tailed random walks. J. Theoret. Prob. 17, 761-768.

[12] KeveI, P. (2009). Linear combinations of i.i.d. random variables from the domain of geometric partial attraction of a semistable law. Doctoral Thesis, Szeged University (in Hungarian).

[13] Martin-Löf, A. (1985). A limit theorem which clarifies the 'Petersburg paradox'. J. Appl. Prob. 22, 634-643.

[14] Stolca, G. (2008). Large gains in the St. Petersburg game. C. R. Acad. Sci. Paris 346, 563-566. 